\section{Comparative acceptance of two milbemycin oxime - praziquantel oral dewormers in dogs from different breeds}

\section{Patricia Ovaert, Marie Varloud, Aurélie Lopez}

Ceva, Libourne, France

\section{INTRODUCTION}

For optimized compliance, the palatability of oral medicinal products is a key parameter. Praziquantel is well-known in human medicine for its bad taste. Palatants are therefore expected to improve the acceptance of praziquantel-based products in dogs. This study compared the voluntary acceptance of a flavoured milbemycin oxime - praziquantel tablet (Milbactor ${ }^{\circledR}$ ) versus an unflavoured one (Milbemax ${ }^{\circledR}$.

\section{METHODS}

This study was performed according to the Guideline on the demonstration of palatability of veterinary medicinal products (CVMP, 2014). 21 healthy dogs ( $8.3 \pm 1.0 \mathrm{~kg}$ body weight; $6.9 \pm$ 3.2 years old) from seven different breeds were allocated to homogenous groups. Each product was offered to each group of dogs on three occasions. The test assessed the individual prehension and consumption of the product. Prehension was assessed as: prehended from bowl within $1 \mathrm{~min}$, prehended from hand within $1 \mathrm{~min}$, or not prehended. Consumption of the product, whether taken from the bowl or from the hand, was assessed as: full, partial or rejected (not ingested or spitted out after prehension). General health observations of dogs were performed on the days of administration. Each product was tested in 63 tests. Statistical comparisons between groups were performed using a Chi-2 test.

\section{RESULTS}

The frequency of prehension of the Milbactor ${ }^{\circledR}$ tablets was higher than the Milbemax ${ }^{\circledR}$ tablets $(p=0.0042)$. Milbactor ${ }^{\circledR}$ tablets were indeed prehended in 42 out of 63 tests $(66.7 \%)$ while Milbemax ${ }^{\circledR}$ tablets were prehended in 26 out of 63 tests $(41.3 \%)$. Out of the Milbactor ${ }^{\circledR}$ tablets that were prehended, $92.9 \%$ were taken successfully (39 tests out of 42).The overall voluntary acceptance rate was improved with the flavoured formulation $(p=0.0044)$. Milbactor ${ }^{\circledR}$ tablets exhibited an overall voluntary acceptance rate of $61.9 \%$ vs. $36.5 \%$ for Milbemax ${ }^{\circledR}$ tablets. Apart from diarrhea observed on a dog given Milbemax ${ }^{\circledR}$ tablet, the dogs tolerated well the products. This study demonstrates that the flavouring agents improved successfully the acceptance of the tablets and contribute therefore to a better compliance in deworming practices of dogs.

\section{Investigations into the effect of feline upper respiratory tract disease on length of stay in a rehoming centre}

\section{Jocelyn Toner ${ }^{1,3}$, Caroline Loach ${ }^{1,3}$, Jenny Stavisky ${ }^{2}$}

Downland Veterinary Group, Hampshire, UK

The University of Nottingham, Nottinghamshire, UK

RSPCA Solent Branch, Hampshire, UK

Feline Upper Respiratory Tract Disease ("FURD", or "Cat flu") is a major problem in rehoming and rescue centres. It is a significant drain on resources and can have a major impact on rehoming. The primary aim of this study was to investigate the relationship between FURD and length of stay for cats entering a shelter. The secondary aim was to investigate whether the risk of FURD differed between stray and relinquished cats entering the shelter.

Data were collected for every cat entering a large rehoming centre in Hampshire consisting of a fluctuating population of approximately 100 cats. Data included origin (relinquished or stray), total length of stay, incidence of clinical signs of FURD, and outcome (euthanasia, return to owner, rehomed or transferred). The case definition for FURD consisted of upper respiratory signs or oral ulceration without or without ocular signs.

Data analysis was performed in SPSS. Data were tested for normality, and continuous data compared using t-test or Mann-Whitney as appropriate. Categorical data were compared using chi-squared. Significance was set at $p<0.05$ throughout.

The final data set consisted of 660 cats. Overall $10.9 \%$ of cats developed signs of FURD during their stay. Cats which developed FURD had a significantly longer stay with a median stay of 28 days for cats without signs of FURD versus 54.5 days for those who did show signs $(p=0.00)$. There was no significant difference in the likelihood of developing FURD between stray and relinquished cats $(p=0.370)$.

The results show that FURD is associated with an increased length of stay. However it is not possible to conclude from this study whether FURD causes the increase, or whether this could be in part due to increased risk of FURD in longer staying cats.

The increase in length of stay has financial implications with each case of FURD costing an average of $f 61.14$ based on a daily cost of caring for a cat and 5 days of antibiotics. An increasing average length of stay also reduces the number of cats which can move through a rehoming centre in a year.

Further investigation is needed to determine whether the majority of cases are from novel infection or recrudescence, which again has huge implications in shelter management and is the focus of our next study. 